\title{
Low frequency terahertz-induced demagnetization in ferromagnetic nickel
}

\author{
Mostafa Shalaby, ${ }^{1, a)}$ Carlo Vicario, ${ }^{1, b)}$ and Christoph P. Hauri ${ }^{1,2, c)}$ \\ ${ }^{1}$ SwissFEL, Paul Scherrer Institute, 5232 Villigen PSI, Switzerland \\ ${ }^{2}$ École Polytechnique Fédérale de Lausanne, 1015 Lausanne, Switzerland
}

(Received 3 February 2016; accepted 20 April 2016; published online 3 May 2016)

\begin{abstract}
A laser stimulus at terahertz $(\mathrm{THz})$ frequency is expected to offer superior control over magnetization dynamics compared to an optical pulse, where ultrafast demagnetization is mediated by heat deposition. As a THz field cycle occurs on a timescale similar to the natural speed of spin motions, this can open a path for triggering precessional magnetization motion and ultimately ultrafast magnetic switching by the THz magnetic field component, without quenching. Here, we explore the ultrafast magnetic response of a ferromagnetic nickel thin film excited by a strong $(33 \mathrm{MV} / \mathrm{cm})$ terahertz transient in non-resonant conditions. While the magnetic laser pulse component induces ultrafast magnetic precessions, we experimentally found that at high pump fluence, the $\mathrm{THz}$ pulse leads to large quenching which dominates the precessional motion by far. Furthermore, degradation of magnetic properties sets in and leads to permanent modifications of the Ni thin film and damage. Published by AIP Publishing. [http://dx.doi.org/10.1063/1.4948472]
\end{abstract}

The observation of laser-induced demagnetization dynamics in the $3 \mathrm{~d}$ ferromagnet nickel by means of near infrared (nIR) laser pulses ${ }^{1}$ initiated extensive studies on ultrafast magnetization dynamics (UMD). ${ }^{2-8}$ The majority of the past studies on UMD relied on the indirect spin excitation via heat deposition in the electronic system. In these experiments, the optical femtosecond laser, typically generated by a Ti:sapphire $\operatorname{system}\left(\lambda_{\mathrm{c}}=800 \mathrm{~nm}, \nu_{\mathrm{c}} \approx 380 \mathrm{THz}=2.6 \mathrm{fs}^{-1}\right)$, gives rise to an ultrafast temperature rise of the electronic and the spin system above the Curie temperature resulting in a decay of the macroscopic spin order. ${ }^{8,9}$ The underlying exchange mechanisms of angular momentum and the ultrafast energy conservation between the driving laser, electrons, spins, and lattice ${ }^{10,11}$ are still debated, and scattering between various (quasi-) particles, ${ }^{12-14}$ coherent effects, ${ }^{9}$ and superdiffusive spin transport are addressed. ${ }^{3}$ It is widely accepted, however, that the commonly observed ultrafast magnetic quenching caused by optical excitation relies primarily on the time-integrated laser energy deposited in the sample. In other words, no direct correlation between the temporal spin motion and the temporal oscillation of the magnetic laser field has been observed. While the magnetic loss of contrast occurs on a sub-picosecond timescale, it takes nanoseconds for the magnetic system to recover, thus orders of magnitude temporally longer than the femtosecond heat stimulus. The carrier frequency of the Ti:sapphire laser, $\nu_{\mathrm{c}}=2.6 \mathrm{fs}^{-1}$, is indeed much higher than the typical timescale of the spin dynamics of $0.1-10 \mathrm{ps},{ }^{15}$ that is, in the 0.1-10 THz frequency range.

By selecting a corresponding stimulus oscillating at terahertz $(\mathrm{THz})$ frequencies, we recently reported on a coherent opto-magnetic interaction which showed the capability to reorient magnetization by the magnetic laser field only, on a sub-picosecond timescale. ${ }^{16}$ The underlying mechanism to drive the spin $\mathbf{S}$ is the Zeeman torque $\mathbf{T} \propto \boldsymbol{S} \times \boldsymbol{B}$ induced by

\footnotetext{
a)most.shalaby@gmail.com

b) carlo.vicario@psi.ch

c) christoph.hauri@psi.ch
}

the oscillating magnetic field component $\mathbf{B}$ of the $\mathrm{THz}$ pulse. ${ }^{16,17}$ The observed THz-induced UMD were fundamentally different from Ref. 1, as the former was governed by coherent precessional spin motion, and without magnetization quenching. The results in Ref. 17 have indicated that a full reversal of the spin order requires magnetic field amplitude of several Tesla. Such intense THz pulses have just recently become available ${ }^{18}$ and their interaction with ferromagnetic systems has not been investigated so far. We mention that precession-induced switching has been reported in a past experiment where a sub- $\mathrm{THz}$ radially polarized magnetic field transient from a relativistic electron beam ${ }^{19}$ is applied to a CoCrPt sample. Unfortunately, this scheme did not allow for time-resolved investigation.

Here, we explore the interaction of an intense $\mathrm{THz}$ electromagnetic pulse with the ferromagnetic thin nickel film through a survey of different $\mathrm{THz}$ field strengths to answer the following fundamental questions: are THz-driven UMD in nickel purely precessional driven, in contrast to optical excitation? ${ }^{1}$ If not, is there a threshold in laser field strength where heat deposition and subsequent demagnetization start playing a role? Can sub-ps magnetic switching by precession be achieved in nickel at $\mathrm{THz}$ frequencies by increasing the laser magnetic field?

For our time-resolved experiment, we excited a $15 \mathrm{~nm}$ thin sputtered nickel film hosted on a Si substrate with our ultra-intense $\mathrm{THz}$ bullet ${ }^{18}$ in a dry air environment (humidity $<0.1 \%$ ). Our excitation pulse, with a maximum strength of $11 \mathrm{~T}(33 \mathrm{MV} / \mathrm{cm})$, is a carrier envelope phase stable $\mathrm{THz}$ field with a 1.5-cycle shape and main spectral contents in the 1-4 THz range (Figs. 1(a) and 1(b)). The $\mathrm{THz}$ stimulus is generated by optical rectification in an organic crystal and focused to a diffraction-limited spot. For more details, refer to Ref. 18. As an experimental scheme to measure the THzinduced UMD, we employed collinear THz-pump/magnetooptical Kerr effect (MOKE) probe with a temporal resolution of $50 \mathrm{fs}$ and with the beam configuration schematically depicted in Fig. 1(c). ${ }^{20}$ The external magnetic field $\mathbf{B}$ (and 
(a)
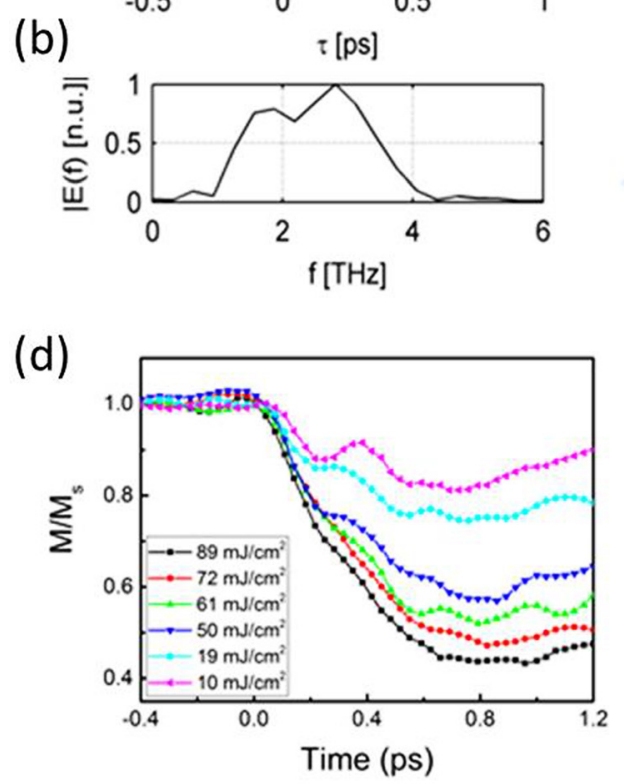

(c)

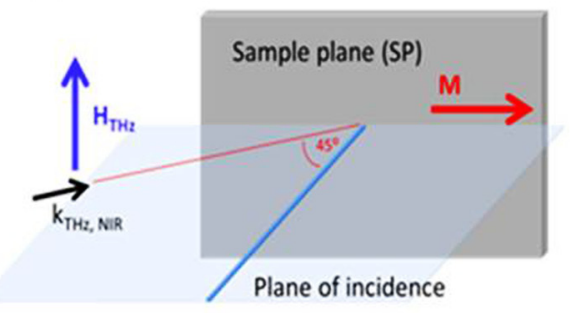

(e)

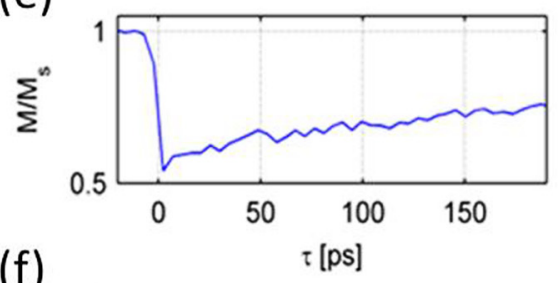

(f)

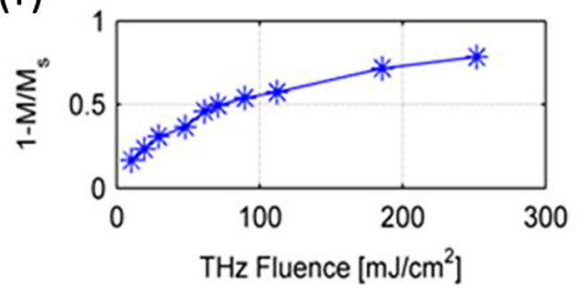

FIG. 1. Terahertz excitation of magnetization dynamics. (a) The 1.5 cycle magnetic field and (b) spectrum of the triggering $\mathrm{THz}$ pulse. (c) $\mathrm{THz}$ and near infrared (nIR) beam configuration on the sample plane together with the magnetization orientation $\mathbf{M}^{20}$ (d) Time-resolved magneto-optical contrast curves for different incidence fluence indicate $\mathrm{THz}$-induced demagnetization. The THz excitation levels used in this set of measurement are 10.5, 19, 50, 61, 72 , and $89 \mathrm{~mJ} / \mathrm{cm}^{2}(2.25,3,4.9,5.4$, 5.88 , and $6.54 \mathrm{~T}$ ). (e) The delayed recovery of the induced demagnetization at a THz pump fluence of $89 \mathrm{~mJ} / \mathrm{cm}^{2}$. (f) The fluence dependence of the peak demagnetization shows a loss of magnetic contrast of almost $80 \%$. thus the magnetization vector $\mathbf{M}$ ) is oriented in the sample plane and parallel to the plane of incidence. The reflected optical probe is analyzed to extract the time-resolved magnetization dynamics. In our experimental setup, the measured MOKE rotation contains information on both the in-plane and out-of-plane magnetization dynamics. In order to eliminate any contributions from the nonmagnetic dynamics, ${ }^{20}$ we modulated the external magnetic field at $25 \mathrm{~Hz}$, which is used as a reference for the lock-in detection system.

Zeeman precession of the spins is induced by the magnetic $\mathrm{THz}$ field component. This coupling mechanism establishes a direct energy transfer from the $\mathrm{THz}$ wave to the spin systems which occurs, in principle, without extensive heating of the electronic system. Neglecting the heating effects, the coherent magnetic precession induced by an external magnetic field can be macroscopically described by LandauLifschitz-Gilbert (LLG) equation. ${ }^{16,17}$ An intense enough $\mathbf{H}^{\mathrm{THz}}$ may induce a time-dependent orientation of $\mathbf{M}$ through $\mathbf{M} \times \mathbf{H}_{\mathrm{eff}}$, where $\mathbf{H}_{\text {eff }}$ is the effective magnetic field including the contributions from the external and $\mathrm{THz}\left(\mathbf{H}^{\mathrm{THz}}\right)$ magnetic fields. On the ultrafast timescale (i.e., on the timescale of the $\mathrm{THz}$ stimulus), the orientation of magnetic vector takes place by ultrafast Zeeman torque which is typically followed by the much slower damping dynamics. ${ }^{17}$ In our experiment, we preserved the configuration $\mathbf{M} \perp\left|\mathbf{H}^{\mathrm{THz}}\right|$ in order to maximize the torque and thus the energy transfer of the opto-magnetic coupling.

In nickel, under low excitation, coherent magnetic precessions are expected to occur similar to the work by Vicario et al. in cobalt ${ }^{16}$ where the amplitude of the induced ultrafast precessions is nearly a linear function of $\mathbf{H}^{\mathrm{THz}} \cdot{ }^{17}$ However, UMD in nickel was found to show only minor coherent motion and only at low flux, governed by our low repetition rate. The quantitative measurements of the THz-induced magnetic dynamics are shown in Fig. 1(d). At even moderate $\mathrm{THz}$ field excitation $\left(>19 \mathrm{~mJ} / \mathrm{cm}^{2}\right)$, the UMD are dominated by ultrafast magnetization quenching. At a maximum field strength of $6.54 \mathrm{~T}$, corresponding to a fluence of $89 \mathrm{~mJ} / \mathrm{cm}^{2}$, the amount of demagnetization reached $58 \%$, corresponding to a loss of magnetic contrast $\mathrm{M} / \mathrm{M}_{\mathrm{sat}}=0.42$. The ultrafast loss of spin order occurs on the scale of $500 \mathrm{fs}$ and coincides with the FWHM temporal duration of the THz envelope. We mention that the observation of such demagnetization induced by a $\mathrm{THz}$ stimulus has not been reported so far. Compared to cobalt, ${ }^{16}$ the THz-induced UMD are dominated by the incoherent demagnetization even at weak fields. This is likely due to the significantly lower Curie temperature (Ni:627 K, Co:1388 K) and due to smaller magnetization at saturation $\mathrm{M}_{\text {sat }}(\mathrm{Ni}: 48.5 \mathrm{emu} / \mathrm{g}, \mathrm{Co}: 143 \mathrm{emu} / \mathrm{g}$ (Ref. 21)). This makes nickel more sensitive to laser-induced temperature changes and consequently to heat-induced demagnetization than cobalt.

Based on a simplified two temperature model and on the heat capacity and coupling of electron and lattice systems, we calculated that the electron temperature in nickel increases up to $860 \mathrm{~K}$ when the maximum flux $\left(89 \mathrm{~mJ} / \mathrm{cm}^{2}\right)$ is applied. ${ }^{1,22}$ A more accurate description of the UMD induced by $\mathrm{THz}$ requires more complex modeling such as Landau-LifshitzBloch, ${ }^{23,24}$ which is beyond the scope of this paper. We believe that the heating of the electronic system might be caused by the large ponderomotive energy that the free charge carriers gain under the influence of the strong co-propagating $\mathrm{THz}$ electric field component resulting in dissipative electronelectron scattering events. As the material is progressively demagnetized, $\mathbf{M}$ is quenched, and the Zeeman torque responsible for the coherent precession becomes weaker.

Figure 1(e) shows the pronounced delayed demagnetization dynamics which extend beyond 180 ps (limited by our 
(a)

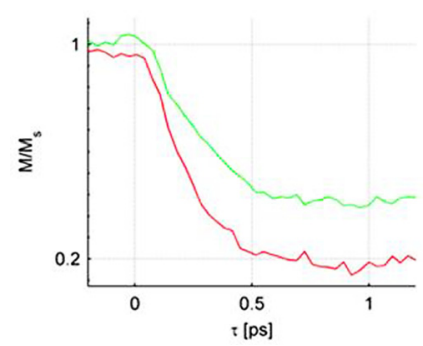

(b)

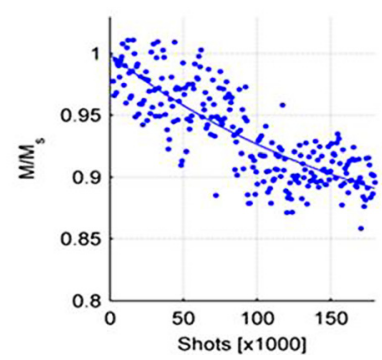

FIG. 2. Terahertz-induced permanent modification on the magnetic properties. (a) The THz-induced magnetization at material damaging fluences of $112 \mathrm{~mJ} / \mathrm{cm}^{2}$ (green) and $252 \mathrm{~mJ} / \mathrm{cm}^{2}$ (red). (b) The degradation in the measured saturation magnetization versus the number of shots for a fixed delay of $-10 \mathrm{ps}$ (before the demagnetization occurs).

delay line range) for $\mathrm{THz}$ fluence of $89 \mathrm{~mJ} / \mathrm{cm}^{2}$. The fluence dependence of demagnetization is shown in Fig. 1(f). At low fluence $\left(<30 \mathrm{~mJ} / \mathrm{cm}^{2}\right)$, the measurements indicate nearly linear dependence. At a higher fluence between 30 and $89 \mathrm{~mJ} /$ $\mathrm{cm}^{2}$, the magnetization dependence starts to deviate from linear showing an onset of saturation.

At even higher excitation fluence $\left(>89 \mathrm{~mJ} / \mathrm{cm}^{2}\right)$, the nickel film starts to permanently lose its magnetic properties and develops surface damage similar to the experiment using conventional laser stimulus. ${ }^{25}$ Fig. 2(a) shows the demagnetization curves at fluences of $112 \mathrm{~mJ} / \mathrm{cm}^{2}$ (green) and $252 \mathrm{~mJ} /$ $\mathrm{cm}^{2}$ (red). At a fluence of $252 \mathrm{~mJ} / \mathrm{cm}^{2}$, the magnetization drops by $80 \%$ with respect to $\mathrm{M}_{\text {sat }}$. Moreover, the initial static magnetization signal (saturation magnetization) reduces progressively with the accumulated $\mathrm{THz}$ dose (Fig. 2(b)). The change of magnetic properties is cumulative and permanent over time. Thus, the demagnetization curve obtained at fluence above $110 \mathrm{~mJ} / \mathrm{cm}^{2}$ depends on the previously accumulated dose. In Fig. 2(b), we measured the

reduction of the saturation magnetization as function of accumulated $\mathrm{THz}$ shots which reached $14 \%$ after 150000 $\mathrm{THz}$ shots. The degradation in the saturation magnetization is recorded at a fixed delay of $-10 \mathrm{ps}$ prior to the strong $\mathrm{THz}$ pulse.

To understand the loss of the magnetic properties, we examined the $\mathrm{Ni}$ film irradiated at the highest $\mathrm{THz}$ fluence with a scanning electron microscope (SEM) (Fig. 3). The analysis revealed that the irradiation with $\mathrm{THz}$ pulse available in the experiment induces permanent damages to the $\mathrm{Ni}$ surface. The SEM images of the irradiated area after 1 and 6000 shots are shown in (a) and (b), respectively. The damaged area (white region) corresponds to the $\mathrm{THz}$ focus size at the sample and the elliptical shape results from the $45^{\circ}$ of incidence. A single $\mathrm{THz}$ shot was found to produce permanent modification on the surface. As the accumulated dose increases, the damaged area becomes larger and part of the film layer is removed. A closer look at Figure 3(c) reveals that after a single $\mathrm{THz}$ shot, the film morphology is modified almost uniformly. However, after 6000 shots (Fig. 3(b)), the magnetic film tends to abrade and develop cracks. In this condition, parts of the nickel are peeled off in several points. The physical mechanism of the damage and the loss of magnetic properties are not investigated here and need further studies. It is likely related to the heating of the film and the effect of the high $\mathrm{THz}$ electric field. However, our experimental results showed that the reduction in the saturation magnetization cannot be completely explained by material loss and by the change of the sample reflectivity.

In conclusion, we performed time-resolved measurements to explore ultrafast magnetization dynamics of a thin nickel film in the previously inaccessible terahertz strongfield regime (1-11 T). We showed heat-induced demagnetization in the ferromagnetic nickel. At a pump fluence of 89 $\mathrm{mJ} / \mathrm{cm}^{2}$, up to $58 \%$ reduction in the magnetic contrast is
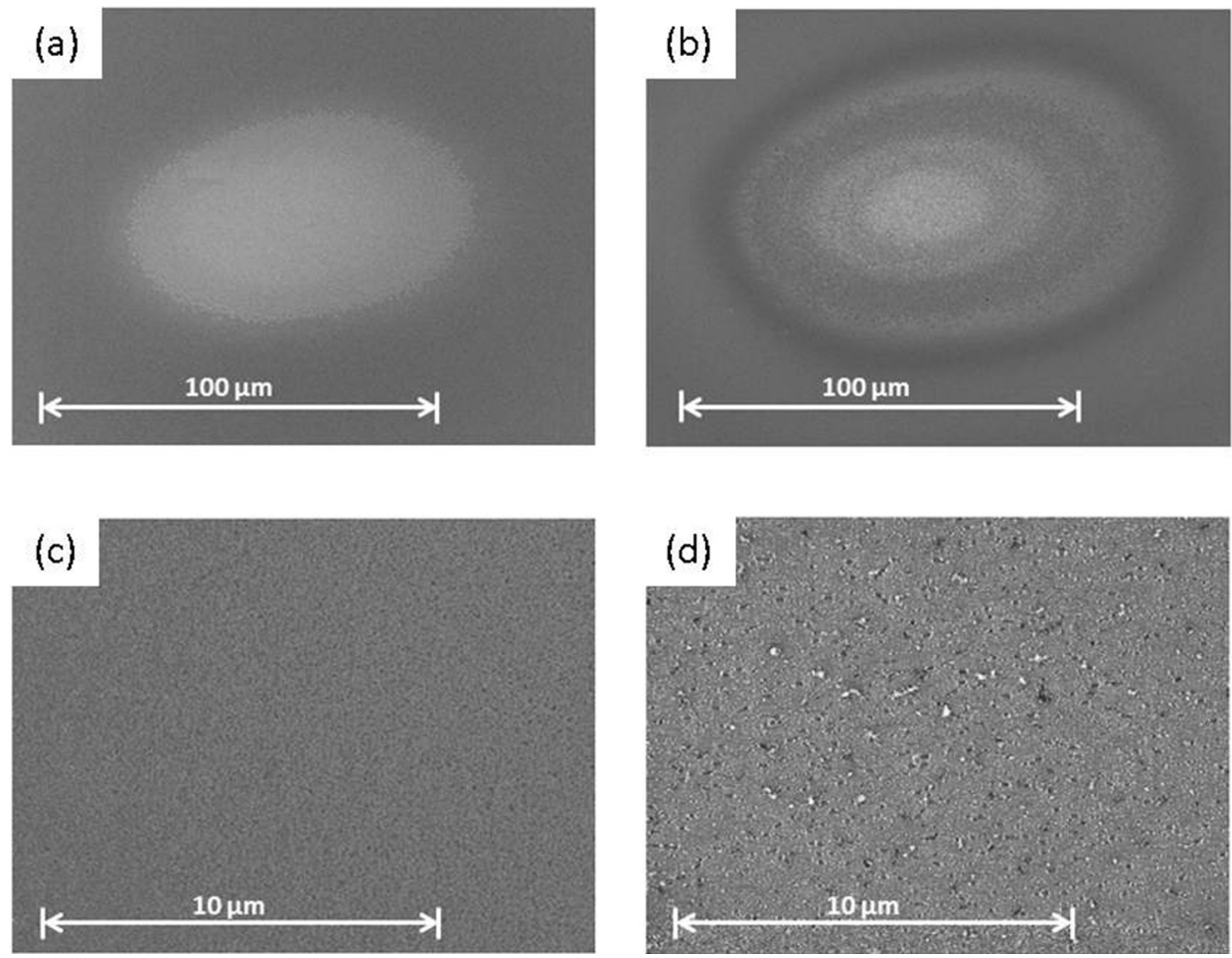

FIG. 3. Scanning electron microscope image of the damaged Ni film illuminated by (a) 1 and (b) 6000 shots at the maximum $\mathrm{THz}$ fluence $\left(252 \mathrm{~mJ} / \mathrm{cm}^{2}\right)$, respectively. (c) and (d) The zoomedin central area of (a) and (b), respectively. 
reached. The incoherent demagnetization occurs on a time scale comparable to that of the terahertz pump, while the recovery time extends over a scale longer than 180 ps. The large incoherent dynamics observed hinder a clear signature of precessional coherent spin control and switching. At THz fluence higher than $89 \mathrm{~mJ} / \mathrm{cm}^{2}$, the THz transient is found to induce permanent loss of the magnetic properties and surface damage visible under the scanning electron microscope. Our experimental results set limitations in coherent control of magnetic moment in nickel and raise questions on the applicability of nickel thin films and other conducting ferromagnets for future ultrafast data storage devices based on precessional switching magnetic bits at $\mathrm{THz}$ speeds.

We acknowledge the support from Edwin Divall in DAQ, Michael Horisberger for sample preparation, and Anja Weber for the SEM characterization. We acknowledge financial support from the Swiss National Science Foundation (SNSF) (Grant No. 200021_146769). M.S. acknowledges partial funding from the European Community's Seventh Framework Programme (FP7/2007-2013) under Grant Agreement No. 290605 (PSI-FELLOW/COFUND). CPH acknowledges association with NCCR-MUST.

${ }^{1}$ E. Beaurepaire, J. C. Merle, A. Daunois, and J. Y. Bigot, "Ultrafast spin dynamics in ferromagnetic nickel," Phys. Rev. Lett. 76, 4250 (1996).

${ }^{2}$ A. J. Schellekens, W. Verhoeven, T. N. Vader, and B. Koopmans, "Investigating the contribution of superdiffusive transport to ultrafast demagnetization of ferromagnetic thin films," Appl. Phys. Lett. 102, 252408 (2013).

${ }^{3}$ M. Battiato, K. Carva, and P. M. Oppeneer, "Superdiffusive spin transport as a mechanism of ultrafast demagnetization," Phys. Rev. Lett. 105, 027203 (2010).

${ }^{4}$ K. Carva, M. Battiato, and P. M. Oppeneer, "Ab initio investigation of the elliott-yafet electron-phonon mechanism in laser-induced ultrafast demagnetization," Phys. Rev. Lett. 107, 207201 (2011).

${ }^{5}$ C. La-O-Vorakiat, E. Turgut, C. A. Teale, H. C. Kapteyn, M. M. Murnane, S. Mathias, M. Aeschlimann, C. M. Schneider, J. M. Shaw, H. T. Nembach et al., "Ultrafast demagnetization measurements using extreme ultraviolet light: Comparison of electronic and magnetic contributions," Phys. Rev. X 2, 011005 (2012).

${ }^{6}$ B. Y. Muller, A. Baral, S. Vollmar, M. Cinchetti, M. Aeschlimann, H. C. Schneider, and B. Rethfeld, "Feedback effect during ultrafast demagnetization dynamics in ferromagnets," Phys. Rev. Lett. 111, 167204 (2013).

${ }^{7}$ B. Koopmans, G. Malinowski, F. Dalla Longa, D. Steiauf, M. Fahnle, T. Roth, M. Cinchetti, and M. Aeschlimann, "Explaining the paradoxical diversity of ultrafast laser-induced demagnetization," Nat Mater. 9, 259 (2010).

${ }^{8}$ T. Roth, A. J. Schellekens, S. Alebrand, O. Schmitt, D. Steil, B. Koopmans, M. Cinchetti, and M. Aeschlimann, "Temperature dependence of laser-induced demagnetization in Ni: A key for identifying the underlying mechanism," Phys. Rev. X 2, 021006 (2012).

${ }^{9}$ J.-Y. Bigot, M. Vomir, and E. Beaurepaire, "Coherent ultrafast magnetism induced by femtosecond laser pulses," Nat. Phys. 5, 515 (2009).

${ }^{10}$ G. P. Zhang, W. Hübner, G. Lefkidis, Y. Bai, and T. F. George, "Paradigm of the time-resolved magneto-optical Kerr effect for femtosecond magnetism," Nat. Phys. 5, 499 (2009).

${ }^{11}$ B. Carva, M. Battiato, and P. Oppeneer, "Is the controversy over femtosecond magneto-optics really solved?," Nat. Phys. 7, 665 (2011).

${ }^{12}$ B. Y. Mueller, T. Roth, M. Cinchetti, M. Aeschlimann, and B. Rethfeld, "Driving force of ultrafast magnetization dynamics," New. J. Phys. 13, 123010 (2011).

${ }^{13}$ B. Koopmans, J. J. M. Ruigrok, F. Dalla Longa, and W. J. M. de Jonge, "Unifying ultrafast magnetization dynamics," Phys. Rev. Lett. 95, 267207 (2005).

${ }^{14}$ E. Carpene, E. Mancini, C. Dallera, M. Brenna, E. Puppin, and S. De Silvestriy, "Dynamics of electron-magnon interaction and ultrafast demagnetization in thin iron films," Phys. Rev. B 78, 174422 (2008).

${ }^{15}$ A. Kirilyuk, A. V. Kimel, and T. Rasing, "Ultrafast optical manipulation of magnetic order," Rev. Mod. Phys. 82, 2731 (2010).

${ }^{16}$ C. Vicario, C. Ruchert, F. Ardana-Lamas, P. M. Derlet, B. Tudu, J. Luning, and C. P. Hauri, "Off-resonant magnetization dynamics phaselocked to an intense phase-stable terahertz transient," Nat. Photonics 7, 720-723 (2013).

${ }^{17}$ M. Shalaby, F. Vidal, M. Peccianti, R. Morandotti, F. Enderli, T. Feurer, and B. D. Patterson, "Terahertz macrospin dynamics in insulating ferrimagnets," Phys. Rev. B 88, 140301(R) (2013).

${ }^{18}$ M. Shalaby and C. P. Hauri, "Demonstration of a low-frequency threedimensional terahertz bullet with extreme brightness," Nat. Commun. 6, 5976 (2015).

${ }^{19}$ I. Tudosa, C. Stamm, A. B. Kashuba, F. King, H. C. Siegmann, J. Stöhr, G. Ju, B. Lu, and D. Weller, "The ultimate speed of magnetic switching in granular recording media," Nature 428, 831 (2004).

${ }^{20}$ M. Shalaby, C. Vicario, and C. P. Hauri, "Simultaneous electronic and the magnetic excitation of a ferromagnet by intense THz pulses," New J. Phys. 18, 013019 (2016).

${ }^{21}$ H. M. Lu, W. T. Zheng, and Q. Jiang, "Saturation magnetization of ferromagnetic and ferromagnetic nanocrystals at room temperature," J. Phys. D: Appl. Phys. 40, 320 (2007).

${ }^{22}$ Z. Lin, L. V. Zhigilei, and V. Celli, "Electron-phonon coupling and electron heat capacity of metals under conditions of strong electron-phonon nonequilibrium," Phys. Rev. B 77, 075133 (2008).

${ }^{23}$ D. A. Garanin, "Fokker-Planck and Landau-Lifshitz-Bloch equations for classical ferromagnets," Phys. Rev. B 55, 3050 (1997).

${ }^{24} \mathrm{~L}$. Xu and S. Zhang, "Self-consistent Bloch equation and Landau-LifshitzBloch equation of ferromagnets: A comparison," J. Appl. Phys. 113, 163911 (2013).

${ }^{25}$ D. Cheskis, A. Porat, L. Szapiro, O. Potashnik, and S. Bar-Ad, "Saturation of the ultrafast laser-induced demagnetization in nickel," Phys. Rev. B 72, 014437 (2005). 